PROCEEDINGS OF THE

AMERICAN MATHEMATICAL SOCIETY

Volume 138, Number 5, May 2010, Pages 1639-1644

S 0002-9939(09)10261-7

Article electronically published on December 16, 2009

\title{
SPECTRAL RADIUS ALGEBRAS AND SHIFT
}

\author{
SRDJAN PETROVIC
}

(Communicated by Marius Junge)

\begin{abstract}
We consider spectral radius algebras associated to operators of the form $h(S)$, where $h \in H^{\infty}$ and $S$ is the unilateral shift. We show that, for a large class of $H^{\infty}$ functions, $\mathcal{B}_{h(S)}$ is weakly dense in $\mathcal{L H}$.
\end{abstract}

In this paper we continue the study of the spectral radius algebras (SRA) initiated in [2. These algebras represent a very interesting class of non-selfadjoint, non-closed operator algebras. Furthermore, as we will see, the relationship between the operator to which an SRA is associated and the algebra itself is highly nonlinear. Finally, they are quite substantial, always containing the commutant of the operator in question.

Let $S$ be the unilateral shift acting on the Hilbert space $\mathcal{H}$. One knows that, whenever $h$ is a function in $H^{\infty}$, the Hardy space of essentially bounded functions on the disk $\mathbb{D}$, one can define the operator $h(S)$. The main object of the present study will be the spectral radius algebras associated with the latter operator.

Before we continue we briefly introduce the relevant concepts and notation. Throughout the paper, $\mathcal{H}$ will be a complex, separable Hilbert space and it will be identified with the Hardy space $H^{2}=H^{2}(\mathbb{D})$ in the usual manner. When $A$ is an operator in $\mathcal{L}(\mathcal{H})$ (the algebra of all bounded linear operators on $\mathcal{H}$ ) with spectral radius $r=r(A)$ and $m$ is a non-negative integer, we define the sequence of positive numbers $d_{m}=m /(1+r m)$ and a sequence of positive, invertible operators $R_{m}=\left(\sum_{m=0}^{\infty} d_{m}^{2 n} A^{* n} A^{n}\right)^{1 / 2}$. The spectral radius algebra $\mathcal{B}_{A}$ is the set $\left\{T \in \mathcal{L}(\mathcal{H}): \sup _{m}\left\|R_{m} T R_{m}^{-1}\right\|<\infty\right\}$. It is not hard to verify that $\mathcal{B}_{A}$ is a unital algebra. The following result from [2] summarizes some of its important properties (cf. [2, Proposition 2.3, Corollary 2.4]).

Proposition 1. Let $A$ be an operator in $\mathcal{L}(\mathcal{H})$. Then $T \in \mathcal{B}_{A}$ if and only if there exists $M>0$ such that, for all $x \in \mathcal{H}$ and $m \in \mathbb{N}, \sum_{n \geq 0} d_{m}^{2 n}\left\|A^{n} T x\right\|^{2} \leq$ $M \sum_{n \geq 0} d_{m}^{2 n}\left\|A^{n} x\right\|^{2}$. When $A T=\lambda T A,|\lambda| \leq 1$, and in particular if $A T=T A$, then $T \in \mathcal{B}_{A}$.

It is easy to see that, when $A$ is the unilateral shift $S$, every operator $T$ satisfies the inequality in Proposition 1] so $\mathcal{B}_{S}=\mathcal{L}(\mathcal{H})$. In fact, more is true, as [1, Theorem 2.7] shows.

Received by the editors February 12, 2009.

2000 Mathematics Subject Classification. Primary 47A65; Secondary 47B15, 47B20.

Key words and phrases. Spectral radius algebras, unilateral shift.

(C)2009 American Mathematical Society Reverts to public domain 28 years from publication 
Theorem 2. Let $A \in \mathcal{L}(\mathcal{H})$. Then $B_{A}=\mathcal{L}(\mathcal{H})$ if and only if the operator $A$ is similar to a constant multiple of an isometry.

Remark 3. It is not hard to verify that, when $A=h(S)$, another equivalent statement can be added to those in Theorem 2, namely, that $h$ is a scalar multiple of an inner function.

Proposition 1 shows that if $\{A\}^{\prime}$ denotes the commutant of $A \in \mathcal{L}(\mathcal{H})$, then

$$
\{A\}^{\prime} \subseteq \mathcal{B}_{A} \subseteq \mathcal{L}(\mathcal{H})
$$

A major problem in the theory of spectral radius algebras is to give a good description of $\mathcal{B}_{A}$ when it is different from either of the two extremes. One line of investigation consists of trying to establish that it possesses a non-trivial invariant subspace (n. i. s.). In view of (0.1) such a subspace is at least hyperinvariant for $A$. This is the case when $A$ is a compact operator (cf. [2, Theorem 4.1]), or belongs to a large class of normal operators (cf. [1, Theorem 3.1]. The following result along these lines (cf. [2, Theorem 3.4]) will be used later in this work. We use the notation $\mathcal{Q}_{A}=\left\{T:\left\|R_{m} T R_{m}^{-1}\right\| \rightarrow 0\right\}$.

Theorem 4. If there is a non-zero compact operator in $\mathcal{Q}_{A}$, then $\mathcal{B}_{A}$ has n. i. s.

When $A=h(S)$, the equality $\mathcal{B}_{A}=\mathcal{L}(\mathcal{H})$ is characterized by Remark 3 , The inclusion $\{A\}^{\prime} \subseteq \mathcal{B}_{A}$ is much less understood. As of this writing, it is not known whether $\{A\}^{\prime}=\mathcal{B}_{A}$ for any non-scalar operator $A$. Equally hard is to establish that $\{A\}^{\prime} \neq \mathcal{B}_{A}$. This has been accomplished in the case when $A$ is a compact operator that is not quasinilpotent 2, Proposition 4.3] or a normal operator [1, Theorem 3.12]. In this paper we will show that even if $h$ is not a scalar multiple of an inner function, $\mathcal{B}_{A}$ can be very different from $\{A\}^{\prime}$. To that end, we notice that a rank one operator $u \otimes v$ commutes with an operator $A$ if and only if $u$ and $v$ are eigenvectors for $A$ and $A^{*}$, respectively. Since the operator of multiplication by an $H^{\infty}$ function $h$ has no eigenvalues, it follows that the commutant of $h(S)$ cannot contain any rank one operators. On the other hand, we will demonstrate that, for a large class of functions $h \in H^{\infty}$, the set of rank one operators in $\mathcal{B}_{h(S)}$ is weakly dense in $\mathcal{L}(\mathcal{H})$. As a result, we obtain a much better insight into the less transparent portion of such an algebra. Perhaps not surprisingly, our results that establish the existence of rank one operators in $\mathcal{B}_{h(S)}$ (see Theorems 8 and 10 below) are based on the boundary behavior of $h$ on the unit circle.

It follows from the definition that $u \otimes v \in \mathcal{B}_{A}$ if and only if $\sup _{m}\left\|R_{m} u\right\|\left\|R_{m}^{-1} v\right\|<$ $\infty$. Notice that $R_{m}^{-1}$ is a contraction, so the last condition is satisfied whenever $\sup _{m}\left\|R_{m} u\right\|$ is finite. Before we can establish a necessary and sufficent condition for the existence of such a vector $u$, we need the following simple result. Since we were unable to find it explicitly stated in the literature, we present it with a brief proof, based on two theorems from [3].

Theorem 5. Let $F$ be a non-negative function on $\mathbb{T}$. Then there exists an outer function $f \in H^{p}, 1 \leq p \leq \infty$, such that $|f|=F$ if and only if $F \in L^{p}$ and $\log F \in L^{1}$.

Proof. If $\log F \in L^{1}$, then one can define an outer function $f$ as

$$
f(z)=\exp \left\{\frac{1}{2 \pi} \int_{0}^{2 \pi} \frac{e^{i t}+z}{e^{i t}-z} \log F\left(e^{i t}\right) d t\right\} .
$$


If, in addition, $F \in L^{p}$, it follows from [3, Theorem 17.16] that $f \in H^{p}$ and $|f|=F$.

Suppose now that $f \in H^{p}$. Clearly $|f| \in L^{p}$ and the fact that $\log |f| \in L^{1}$ is a part of [3, Theorem 17.17].

Now we characterize the existence of $f$ such that $\sup _{m}\left\|R_{m} f\right\|<\infty$.

Theorem 6. Let $h \in H^{\infty}$, let $M=\|h\|_{\infty}$, and let $R_{m}=R_{m}(h(S))$. Then there exists $f \in H^{2}$ such that $\sup _{m}\left\|R_{m} f\right\|<\infty$ if and only if $\log \left(1-|h|^{2} / M^{2}\right) \in L^{1}(\mathbb{T})$. In this situation $f$ is always of the form $f=g w$, where $g$ is an outer function in $H^{\infty}$ that satisfies $|g|^{2}=1-|h|^{2} / M^{2}$ and $w$ is an arbitrary function in $H^{2}$.

Proof. Suppose that $f \in H^{2}$ and $\sup _{m}\left\|R_{m} f\right\|<\infty$. Notice that

$$
\left\|R_{m} f\right\|^{2}=\sum_{n} d_{m}^{2 n}\left\|h(S)^{n} f\right\|^{2}=\sum_{n} d_{m}^{2 n} \frac{1}{2 \pi} \int_{0}^{2 \pi}\left|h\left(e^{i t}\right)\right|^{2 n}\left|f\left(e^{i t}\right)\right|^{2} d t .
$$

Consequently, if $f=f_{1} f_{2}$ is the inner-outer factorization, then $\left\|R_{m} f\right\|=\left\|R_{m} f_{2}\right\|$, so we can assume that $f$ is an outer function, $f=e^{\varphi}$ with the real part of $\varphi$ in $L^{1}$. Further, by the Monotone Convergence Theorem,

$$
\left\|R_{m} f\right\|^{2}=\frac{1}{2 \pi} \int_{0}^{2 \pi} \frac{\left|f\left(e^{i t}\right)\right|^{2}}{1-d_{m}^{2}\left|h\left(e^{i t}\right)\right|^{2}} d t
$$

so the sequence

$$
\frac{1}{2 \pi} \int_{0}^{2 \pi} \frac{\left|f\left(e^{i t}\right)\right|^{2}}{1-d_{m}^{2}\left|h\left(e^{i t}\right)\right|^{2}} d t, \quad m=0,1,2, \ldots
$$

is bounded. Another application of the Monotone Convergence Theorem now shows that

$$
\frac{\left|f\left(e^{i t}\right)\right|^{2}}{1-\left|h\left(e^{i t}\right)\right|^{2} / M^{2}} \in L^{1} .
$$

Suppose now that $\log \left(1-|h|^{2} / M^{2}\right) \notin L^{1}$. Then $1-|h|^{2} / M^{2}=e^{\psi}$ for some $\psi \notin L^{1}$. It follows from (0.3) that $e^{2 \operatorname{Re} \varphi-\psi} \in L^{1}$ and, by Jensen's Inequality, there exists $K>0$ so that $\int_{0}^{2 \pi}\left[2 \operatorname{Re}\left\{\varphi\left(e^{i t}\right)\right\}-\psi\left(e^{i t}\right)\right] d t \leq K$. Since $\int_{0}^{2 \pi}-\psi\left(e^{i t}\right) d t$ diverges to $+\infty$ it would follow that $\int_{0}^{2 \pi} 2 \operatorname{Re}\left\{\varphi\left(e^{i t}\right)\right\} d t$ diverges to $-\infty$, contradicting the assumption that the real part of $\varphi$ is in $L^{1}$. Therefore, $\log \left(1-|h|^{2} / M^{2}\right) \in L^{1}(\mathbb{T})$.

In the opposite direction, if $\log \left(1-|h|^{2} / M^{2}\right) \in L^{1}(\mathbb{T})$, then, by Theorem 5 , there exists an outer function $g \in H^{\infty}$ such that $1-|h|^{2} / M^{2}=|g|^{2}$. Let $f=g w$ for some $w \in H^{2}$. Then, using (0.2),

$$
\left\|R_{m} f\right\|^{2}=\frac{1}{2 \pi} \int_{0}^{2 \pi} \frac{\left|g\left(e^{i t}\right)\right|^{2}\left|w\left(e^{i t}\right)\right|^{2}}{1-d_{m}^{2}\left|h\left(e^{i t}\right)\right|^{2}} d t \leq \frac{1}{2 \pi} \int_{0}^{2 \pi}\left|w\left(e^{i t}\right)\right|^{2} d t
$$

so $w \in H^{2}$ implies that $\sup _{m}\left\|R_{m} f\right\|$ is finite.

Theorem [6 has an important consequence.

Theorem 7. If $\log \left(1-|h|^{2} / M^{2}\right) \in L^{1}(\mathbb{T})$, then $\mathcal{B}_{h(S)}$ is weakly dense in $\mathcal{L}(\mathcal{H})$.

Proof. With the notation of Theorem 6, $\mathcal{B}_{h(S)}$ contains an operator $f \otimes v$ whenever $f$ belongs to the subspace $g H^{2}$. This subspace is dense in $H^{2}$ (since $g$ is outer), whence the weak closure of $\mathcal{B}_{h(S)}$ contains all rank one operators and, hence, equals $\mathcal{L}(\mathcal{H})$ 
If $f \in H^{2}$ and $\sup _{m}\left\|R_{m} f\right\|$ is finite, then any rank one operator $f \otimes g$ belongs to $\mathcal{B}_{h(S)}$. It is natural to ask whether there are other rank one operators in $\mathcal{B}_{h(S)}$; i.e., is it possible that $\left\|R_{m} u\right\| \rightarrow \infty$ but $\sup _{m}\left\|R_{m} u\right\|\left\|R_{m}^{-1} v\right\|<\infty$ ? The following result shows that, assuming the logarithmic condition above, the answer is negative.

Theorem 8. Suppose that $\log \left(1-|h|^{2} / M^{2}\right) \in L^{1}(\mathbb{T})$. Then a rank one operator $f \otimes g$ belongs to $\mathcal{B}_{h(S)}$ if and only if $\sup _{m}\left\|R_{m} f\right\|<\infty$.

Proof. If $\sup _{m}\left\|R_{m} f\right\|<\infty$ the result follows from the fact that $R_{m}^{-1}$ is a contraction. In the opposite direction, if $f \otimes g \in \mathcal{B}_{h(S)}$ and $\left\|R_{m} f\right\| \rightarrow \infty$, then $\left\|R_{m}^{-1} g\right\| \rightarrow 0$. However, Theorem 6 implies that there is $u \in H^{2}$ such that $\sup _{m}\left\|R_{m} u\right\|<\infty$, so $\left\|R_{m}(u \otimes g) R_{m}^{-1}\right\| \rightarrow 0$. This means that $u \otimes g \in \mathcal{Q}_{h(S)}$ and, by Theorem 4 , $\mathcal{B}_{h(S)}$ has n. i. s., which contradicts Theorem 7 .

When $\log \left(1-|h|^{2} / M^{2}\right) \in L^{1}(\mathbb{T})$, Theorems 6 and 8 give a complete characterization of rank one operators in $\mathcal{B}_{h(S)}$. Namely, in the situation, a rank one operator $u \otimes v$ belongs to $\mathcal{B}_{h(S)}$ if and only if $u \in g H^{2}$, where $g$ is an outer function such that $|g|^{2}=1-|h|^{2} / M^{2}$. It is easy to see that if $h_{1}, h_{2} \in H^{\infty}$, then $\mathcal{B}_{h_{1}(S)}=\mathcal{B}_{h_{2}(S)}$ if there exists $c>0$ such that $\left|h_{1}\left(e^{i t}\right)\right|=c\left|h_{2}\left(e^{i t}\right)\right|$, a.e. However, as our next example shows, the algebras $\mathcal{B}_{h_{1}(S)}$ and $\mathcal{B}_{h_{2}(S)}$ can be equal even without the last condition.

Example 9. Let $h_{1}(z)=(z+4) / 5, h_{2}(z)=(z+1)(3 z+2) / 10$. Then $d_{m}\left(h_{1}\right)=$ $d_{m}\left(h_{2}\right)=m /(m+1)$ and a calculation shows that

$$
\frac{1-d_{m}^{2}\left|h_{1}\left(e^{i t}\right)\right|^{2}}{1-d_{m}^{2}\left|h_{2}\left(e^{i t}\right)\right|^{2}}=\frac{(1-\cos t) 32 m^{2}+100(2 m+1)}{(1-\cos t)\left(24 m^{2} \cos t+74 m^{2}\right)+100(2 m+1)} .
$$

Notice that $24 m^{2} \cos t+74 m^{2} \geq-24 m^{2}+74 m^{2} \geq 32 m^{2}$, so

$$
\frac{1-d_{m}^{2}\left|h_{1}\left(e^{i t}\right)\right|^{2}}{1-d_{m}^{2}\left|h_{2}\left(e^{i t}\right)\right|^{2}} \leq 1
$$

On the other hand,

$$
\begin{aligned}
\frac{(1-\cos t)\left(24 m^{2} \cos t+74 m^{2}\right)+100(2 m+1)}{(1-\cos t) 32 m^{2}+100(2 m+1)} \\
\quad=\frac{(1-\cos t)\left(24 m^{2} \cos t+74 m^{2}\right)}{(1-\cos t) 32 m^{2}+100(2 m+1)}+\frac{100(2 m+1)}{(1-\cos t) 32 m^{2}+100(2 m+1)} \\
\quad \leq \frac{24 \cos t+74}{32}+1 \leq 5,
\end{aligned}
$$

SO

$$
\frac{1-d_{m}^{2}\left|h_{1}\left(e^{i t}\right)\right|^{2}}{1-d_{m}^{2}\left|h_{2}\left(e^{i t}\right)\right|^{2}} \geq 1 / 5
$$

Let $Y$ be an operator in $\mathcal{B}_{h_{1}(S)}$. By Proposition 1, there exists $K>0$ such that, for any $f \in H^{2}$ and $m \in \mathbb{N}$,

$$
\int_{0}^{2 \pi} \frac{\left|(Y f)\left(e^{i t}\right)\right|^{2}}{1-d_{m}^{2}\left|h_{1}\left(e^{i t}\right)\right|^{2}} d t \leq K \int_{0}^{2 \pi} \frac{\left|f\left(e^{i t}\right)\right|^{2}}{1-d_{m}^{2}\left|h_{1}\left(e^{i t}\right)\right|^{2}} d t .
$$

Using (0.4) and (0.5) we obtain that

$$
\int_{0}^{2 \pi} \frac{\left|(Y f)\left(e^{i t}\right)\right|^{2}}{1-d_{m}^{2}\left|h_{2}\left(e^{i t}\right)\right|^{2}} d t \leq \frac{K}{5} \int_{0}^{2 \pi} \frac{\left|f\left(e^{i t}\right)\right|^{2}}{1-d_{m}^{2}\left|h_{2}\left(e^{i t}\right)\right|^{2}} d t
$$


and it follows that $Y \in \mathcal{B}_{h_{2}(S)}$. The other inclusion can be established in the same way, so $\mathcal{B}_{h_{1}(S)}=\mathcal{B}_{h_{2}(S)}$.

Next, we turn our attention to the case when $\log \left(1-|h|^{2} / M^{2}\right) \notin L^{1}(\mathbb{T})$. Clearly, this happens whenever $|h|$ attains its maximum $M$ on a set $B$ of positive measure. If $B=\mathbb{T}$, then $h$ is a scalar multiple of an inner function, and Remark 3 shows that $\mathcal{B}_{h(S)}=\mathcal{L}(\mathcal{H})$. Another possibility is that $\mathbb{T}=A \cup B$, both $A$ and $B$ have positive measure, and $|h|=M$ on $B,|h|<M$ on $A$. Once again, we will show that $\mathcal{B}_{h(S)}$ contains a dense set of rank one operators.

Theorem 10. Suppose that $\mathbb{T}=A \cup B$, both $A$ and $B$ have positive measure, and $h$ is an $H^{\infty}$ function satisfying $|h|=M$ on $B,|h|<M$ on $A$. Then $\mathcal{B}_{h(S)}$ is weakly dense in $\mathcal{L}(\mathcal{H})$.

Proof. Let $w \in L^{2}(B)$ and let $v=P_{H^{2}} w$ (the projection of $w$ on $H^{2}$ identified with the subspace $H^{2} \oplus(0)$ of $\left.L^{2}\right)$. We will show that $u \otimes v \in \mathcal{B}_{h(S)}$ for any $u \in H^{2}$.

Let $h_{m}$ denote the $L^{2}$ function $\sqrt{1 /\left(1-d_{m}^{2}|h|^{2}\right)}$. With $R_{m}=R_{m}(h(S))$, (0.2) implies that, for any $f \in H^{2},\left\|R_{m} f\right\|_{H^{2}}=\left\|h_{m} f\right\|_{L^{2}}$. In particular, for any $f \in H^{2}$, $(0.6)$

$$
\left\|R_{m}(u \otimes v) f\right\|_{H^{2}}^{2}=\left\|h_{m}\langle f, v\rangle u\right\|_{L^{2}}^{2}=\int_{A}\left|h_{m}\right|^{2}|\langle f, v\rangle|^{2}|u|^{2}+\int_{B}\left|h_{m}\right|^{2}|\langle f, v\rangle|^{2}|u|^{2} .
$$

Notice that $h_{m}$ is constant on $B$, say $h_{m}=c_{m}>0$. Therefore, the second integral in (0.6) equals $\int_{B}\left|c_{m}\right|^{2}|\langle f, v\rangle|^{2}|u|^{2}=\left|\left\langle c_{m} f, v\right\rangle\right|^{2} \int_{B}|u|^{2} \leq\left|\left\langle c_{m} f, v\right\rangle\right|^{2}\|u\|^{2}$. Further, $\left\langle c_{m} f, v\right\rangle=\left\langle c_{m} f, w\right\rangle=\int_{B} c_{m} f \bar{w}$, so $\left|\left\langle c_{m} f, v\right\rangle\right|^{2} \leq \int_{B}\left|c_{m} f\right|^{2} \int_{B}|w|^{2}=$ $\|w\|^{2} \int_{B}\left|h_{m} f\right|^{2} \leq\left\|R_{m} f\right\|^{2}\|w\|^{2}$. Thus,

$$
\int_{B}\left|h_{m}\right|^{2}|\langle f, v\rangle|^{2}|u|^{2} \leq\left\|R_{m} f\right\|^{2}\|w\|^{2}\|u\|^{2} .
$$

To estimate the first integral in (0.6) we notice that on $A, h_{m} \leq c_{m}$. Therefore,

$$
\begin{aligned}
\int_{A}\left|h_{m}\right|^{2}|\langle f, v\rangle|^{2}|u|^{2} & \leq\left|\left\langle c_{m} f, v\right\rangle\right|^{2} \int_{A}|u|^{2} \leq\left|\left\langle c_{m} f, w\right\rangle\right|^{2}\|u\|^{2} \\
& \leq \int_{B}\left|c_{m} f\right|^{2} \int_{B}|w|^{2}\|u\|^{2} \leq\left\|R_{m} f\right\|^{2}\|w\|^{2}\|u\|^{2} .
\end{aligned}
$$

Combining (0.6), (0.7), and (0.8) now yields

$$
\left\|R_{m}(u \otimes v) f\right\|^{2} \leq 2\|u\|^{2}\|w\|^{2}\left\|R_{m} f\right\|^{2}
$$

and $u \otimes v \in \mathcal{B}_{h(S)}$.

Finally, it is obvious that $P_{H^{2}}\left(L^{2}(B)\right)$ is dense in $H^{2}$, so the operators of the form $u \otimes v$ (with $u \in H^{2}, v \in P_{H^{2}}\left(L^{2}(B)\right)$ ) are dense in the set of all rank one operators and thus $\mathcal{B}_{h(S)}$ is dense.

Unfortunately, in the case when $|h|=M$ on a set of measure 0 and $\log \left(1-|h|^{2} / M^{2}\right) \notin L^{1}(\mathbb{T})$, we were unable to establish the existence of rank one operators in $\mathcal{B}_{h(S)}$. Therefore, we leave it as an open question.

Problem 11. Let $\left|h\left(e^{i t}\right)\right|<M$ a.e. and suppose that $\log \left(1-|h|^{2} / M^{2}\right) \notin L^{1}(\mathbb{T})$. Does the algebra $\mathcal{B}_{h(S)}$ always contain a non-zero rank one operator?

We offer a partial answer to Problem 11] namely in the case when $h$ is analytic on $\mathbb{D}^{-}$. 
Theorem 12. Let $h$ be an analytic function in $\mathbb{D}^{-}$and let $M=\sup \{|h(z)|:|z|=$ 1\}. Then either $h / M$ is an inner function or $\log \left(1-|h|^{2} / M^{2}\right) \in L^{1}(\mathbb{T})$. Either way, $\mathcal{B}_{h(S)}$ contains a dense set of rank one operators.

Proof. Suppose that $h / M$ is not an inner function and that the set $A=\{z:|z|=$ $1,|f(z)|=M\}$ is infinite. Then $A$ must have an accumulation point $z_{0}=e^{i t_{0}}$. Clearly, $\left|h\left(z_{0}\right)\right|=M \neq 0$ so there exists $\epsilon>0$ such that for $\left|t-t_{0}\right|<\epsilon, 1-|h|^{2} / M^{2}$ is an infinitely differentiable function of $t$. If $\sum_{k \geq 0} c_{k}\left(t-t_{0}\right)^{k}$ is its Taylor series, then $c_{0}=0$, so there exist $m \in \mathbb{N}$ and $c>0$ such that $1-|h|^{2} / M^{2}=\left|t-t_{0}\right|^{m}|p(t)| \geq$ $c\left|t-t_{0}\right|^{m}$ for $\left|t-t_{0}\right|<\epsilon$. Consequently, $1-|h|^{2} / M^{2} \neq 0$ for $0<\left|t-t_{0}\right|<\epsilon$, contradicting the fact that $z_{0}$ is an accumulation point of $A$.

Thus, if $h / M$ is not an inner function, the set $A$ must be finite, $A=\left\{e^{i t_{1}}, e^{i t_{2}}, \ldots\right.$, $\left.e^{i t_{n}}\right\}$. As already noted, if $1 \leq k \leq n$, there exists $\epsilon_{k}>0$ such that for $\left|t-t_{k}\right|<\epsilon_{k}$, $\log \left(1-\left|h\left(e^{i t}\right)\right|^{2} / M^{2}\right) \geq \log c+m \log \left|t-t_{k}\right|$. Since it is well known that $\int_{t_{k}-\epsilon_{k}}^{t_{k}+\epsilon_{k}} \log \mid t-$ $t_{k} \mid d t$ converges, the theorem is proved.

\section{REFERENCES}

[1] A. Biswas, A. Lambert, and S. Petrovic, On spectral radius algebras and normal operators, Indiana Univ. Math. J. 56 (2007), no. 4, 1661-1674. MR.2354695 (2008i:47039)

[2] A. Lambert, S. Petrovic, Beyond hyperinvariance for compact operators. J. Funct. Anal. 219 (2005), no. 1, 93-108. MR2108360 (2005i:47028)

[3] W. Rudin, Real and complex analysis. Third edition. McGraw-Hill Book Co., New York, 1987. MR924157 (88k:00002)

Department of Mathematics, Western Michigan University, Kalamazoo, Michigan 49008

E-mail address: srdjan.petrovic@wmich.edu. 